\title{
The use of submucosal small intestinal mesh in laparoscopic sacrocolpopexy and posterior vaginal repair
}

Published online: 12 August 2005

(c) Springer-Verlag Berlin / Heidelberg 2005

\begin{abstract}
Sacrocolpopexy for vaginal vault prolapse changes the mechanical axis of the vagina and can result in prolapse of the anterior or posterior vaginal walls. Thirteen consecutive patients were examined before and after surgery using the POP-Q International Continence Society scoring system for genital prolapse. One patient had an intact cervix and therefore underwent sacrocervicopexy, whereas two patients had sacrohysteropexy. The other patients had sacrocolpopexy. Porcine small intestinal submucosal (SIS) absorbable mesh (Surgisis, Cook) was fixed to the posterior vaginal wall. The posterior wall remained well supported following SIS mesh interposition between the posterior vaginal wall and the rectum. Meshes can provide the much-needed support in pelvic reconstruction.
\end{abstract}

Keywords Vaginal prolapse - Pelvic floor - Surgery · Laparoscopy

\section{Introduction}

We present thirteen consecutive patients who were examined before and after surgery using the POP-Q International Continence Society scoring system for genital prolapse [1]. Vaginal vault prolapse occurs in up to $11.6 \%$ of patients who undergo hysterectomy for genital prolapse $[2,3]$. Inherent connective tissue weakness is a common finding in women with genital prolapse

A. Cutner $(\bowtie)$

Department of Obstetrics and Gynaecology,

University College London Hospital, Huntley Street,

London, WC1E 6HX, UK

E-mail: alfred@cutner.freeserve.co.uk

\section{Spiteri}

Department of Obstetrics and Gynaecology,

Chelsea and Westminster Hospital,

Fulham Road, SW1, UK

E-mail: mspiteri@onetel.com
$[4,5]$, and this might explain the increased risk of recurrence in some women. There are often multiple defects, with combined prolapse in any of the three different compartments of the pelvic floor [6]. Treatment of concomitant anterior and posterior wall fascial defects at the time of abdominal open or laparoscopic sacrocolpopexy using synthetic meshes has been described [7,8].

Similarly, following initial vault repair, a change in mechanical axis of the vagina can result in prolapse of the anterior or posterior vaginal walls [9].

\section{Objectives}

The aim of this study was to determine whether laparoscopic insertion of porcine small intestinal submucosal (SIS) absorbable mesh (Surgisis, Cook) on the lower posterior vaginal wall prevents posterior wall prolapse after laparoscopic sacrocolpopexy.

\section{Methodology}

Consecutive patients between December 2000 and November 2001 who underwent laparoscopic prolapse repair of the vaginal vault together with posterior vaginal wall repair were recruited for this prospective observational study. One patient had an intact cervix and therefore underwent sacrocervicopexy, whereas two patients had sacrohysteropexy. Three patients underwent concomitant colposuspension to correct urinary urodynamic stress incontinence.

All patients were examined before and after surgery using the POP-Q International Continence Society scoring system for genital prolapse [10]. They were followed up at 6,12 , and 24 weeks after surgery.

Table 1 shows the pelvic floor surgery that our patients had undergone prior to this intervention. For five patients, this was their third pelvic floor surgery, whereas for two patients this was their fourth surgery. 
Table 1 Previous surgery in patients in this study ( $L A V H$ laparoscopically-assisted vaginal hysterectomy)

\begin{tabular}{lll}
\hline First surgery & Second surgery & Third surgery \\
\hline $\begin{array}{l}\text { Vaginal hysterectomy and anteroposterior repair - 4 } \\
\text { LAVH and anterior repair - 1 } \\
\begin{array}{l}\text { Total abdominal hysterectomy - 5 } \\
\text { Subtotal hysterectomy - 1 }\end{array}\end{array}$ & Anterior and enterocoele repair - 1 & Anterior repair - 1 \\
$\begin{array}{l}\text { Colposuspension - 1 } \\
\text { Manchester repair - 1 }\end{array}$ & Anterior repair - 3 & Colposuspension - 1 \\
\hline
\end{tabular}

\section{Technique}

The patient is placed in the lithotomy position. After catheterisation of the bladder and an adequate pneumoperitoneum, a 10-mm laparoscope is introduced through the umbilicus. Two secondary $5-\mathrm{mm}$ ports are inserted, one at the same level lateral to the rectus sheath on the left-hand side and one on the right-hand side. The fourth port is $10-12 \mathrm{~mm}$ and placed in the midline in the suprapubic region. An assistant inserts a rectal probe in the vagina to exert pressure on the vault from below, along the natural axis of the vagina. A further rectal probe is placed in the rectum so the rectovaginal septum can be easily identified.

The abdominal cavity is inspected, and any adhesions are lysed. The pouch of Douglas and the ureters are well visualised. The peritoneum covering the vault is incised transversely, and the white vaginal fascia is exposed (Fig. 1). Posterior vaginal wall dissection is performed as far down as the perineal body, taking care to avoid damage to the rectum. The SIS mesh is fixed to the posterior vaginal wall using polydioxanone (PDS; Ethicon, Johnson \& Johnson) sutures at its four angles. We speculate that using this mesh rather than a nonabsorbable mesh on the lower part of the vaginal wall will decrease the long-term complications of mesh erosion into adjacent viscera. The mesh is then covered with posterior wall fascia that is brought together from both sides into the midline.

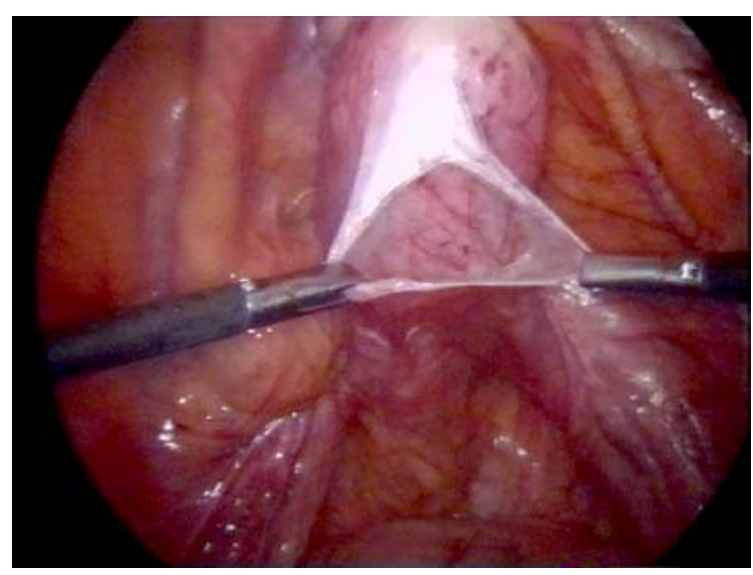

Fig. 1 Dissection of the peritoneum over the vaginal vault
The synthetic prolene mesh (Ethicon, Somerville, NJ) is then cut to fit the entire width of the vaginal apex and is anchored with nonabsorbable polydioxanone (PDS; Ethicon, Johnson \& Johnson) sutures (Fig. 2). Securing the mesh well onto the vagina and avoiding suture pullthrough affects both short- and long-term outcomes. Further sutures fix the mesh at least to the upper half of the vaginal wall posteriorly.

The presacral space is then entered as the sigmoid colon is reflected towards the left pelvic sidewall. The sacral promontory is palpated with the laparoscopic grasping forceps, and the presacral vessels are identified. The peritoneum overlying the sacral promontory is then incised longitudinally. This is done with great care to avoid the bifurcation of the aorta above, the common iliac vessels and ureters laterally, and the mesentery of the sigmoid colon to the left. A laparoscopic dissector is used to expose the anterior longitudinal ligament of the sacrum. The surgeon then staples the synthetic mesh with titanium spirals, having checked vaginally the right tension of the mesh (Fig. 1). Using tacks is our usual practice because it decreases operating time significantly. Care is taken to avoid excessive tension, but the mesh must be tight enough to provide adequate support when the patient is standing. We do not bury the mesh. There is no evidence in the literature that peritonisation has any advantages.

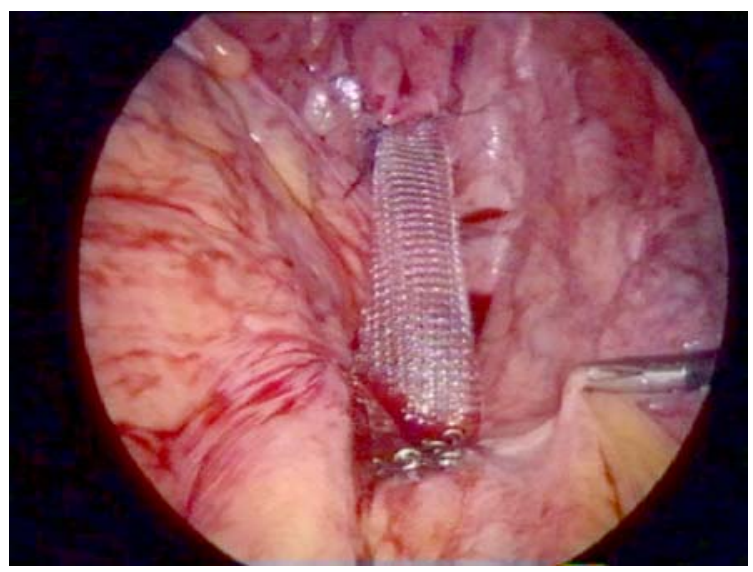

Fig. 2 End result showing the polypropylene-prolene mesh suspending the vault to the sacrum 


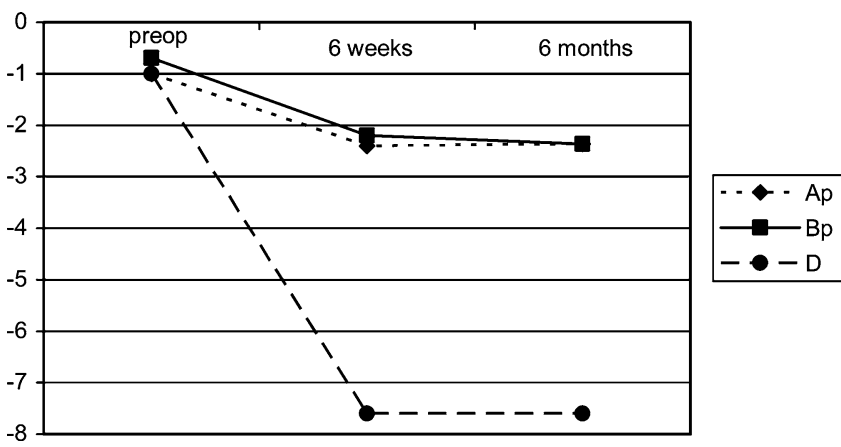

Fig. 3 POP-Q examination findings before and after surgery

\section{Results}

Figure 3 shows a graphic representation of the results. $\mathrm{Ap}, \mathrm{Bp}$, and $\mathrm{D}$, according to the POP-Q score, are, respectively, a point of the posterior vaginal wall, usually $3 \mathrm{~cm}$ proximal to the introitus; the lowest point on the posterior wall of the vagina; and the posterior fornix, usually about $10 \mathrm{~cm}$ from the introitus [11]. Preoperatively, $\mathrm{Bp}$ had a mean value of $0.6 \mathrm{~cm}$, whereas 6 months after surgery the mean value was $2.4 \mathrm{~cm}$. The posterior wall remained well supported following SIS mesh interposition between the posterior vaginal wall and the rectum.

There were no preoperative or immediate postoperative complications. One patient developed recurrent vault prolapse and required repeat surgery 3 months after the first procedure. The posterior wall, however, remained well supported. There were no mesh-related postoperative complications.

\section{Conclusion}

A SIS mesh prevents rectocoele formation following laparoscopic sacrocolpopexy with posterior wall repair. Long-term follow-up of these patients is mandatory. At 1-year follow-up of six patients, the results are consistent.

It is possible that the approximation of the rectovaginal fascia during surgery is enough to prevent rectocoele formation. It is therefore desirable to perform a randomised controlled trial of women undergoing surgery with and without the mesh. Functional assessment of bowel, sexual, and urinary function using standardised questionnaires is also desirable together with the anatomical assessment to investigate any differences related to the mesh. Meshes can provide the much-needed support in pelvic reconstruction. The ideal material has yet to be developed, but this natural absorbable mesh used for laparoscopic repair shows promising results with the possibility of fewer complications.

This small series of laparoscopic colpoperineopexy using a natural absorbable mesh to correct the posterior vaginal defect shows optimum short-term anatomical and functional results. Long-term follow-up is necessary with a larger number of patients to establish whether this natural absorbable mesh has fewer long-term complications with similar or better functional results.

\section{References}

1. Bump RC, Mattiasson A, Bo K, Brubaker LP, De Lancey JO, Klarskov $P$ et al (1996) The standardization of terminology of female pelvic organ prolapse and pelvic floor dysfunction. Am J Obstet Gynecol 175(1):10-17

2. Marchionni M, Bracco GL, Checcucci V, Carabaneanu A, Coccia EM, Mecacci $F$ et al (1999) True incidence of vaginal vault prolapse. Thirteen years of experience. J Reprod Med 44(8):679-684

3. Mant J, Painter R, Vessey M (1997) Epidemiology of genital prolapse: observations from the Oxford Family Planning Association Study. Br J Obstet Gynaecol 104(5):579-585

4. Norton PA, Baker JE, Sharp HC, Warenski JC (1995) Genitourinary prolapse and joint hypermobility in women. Obstet Gynecol 85(2):225-228

5. Jackson SR, Avery NC, Tarlton JF, Eckford SD, Abrams P, Bailey AJ (1996) Changes in metabolism of collagen in genitourinary prolapse. Lancet 15(347):1658-1661

6. Scotti RJ (1991) Repair of genitourinary prolapse in women. Curr Opin Obstet Gynecol 3(3):404-412

7. Fox SD, Stanton SL (2000) Vault prolapse and rectocele: assessment of repair using sacrocolpopexy with mesh interposition. BJOG 107(11):1371-1375

8. Lyons TL, Winer WK (1997) Laparoscopic rectocele repair using polyglactin mesh. J Am Assoc Gynecol Laparosc 4(3):381-384

9. Baessler KSB (2001) Abdominal sacrocolpopexy and anatomy and function of the posterior compartment. Obstet Gynecol 97(5 Pt 1):678-684

10. Bump RC, Mattiasson A, Bo K, Brubaker LP, De Lancey JO, Klarskov P, Shull BL, Smith AR (1996) The standardization of terminology of female pelvic organ prolapse and pelvic floor dysfunction. Am J Obstet Gynecol 175(1):10-17

11. Bump RC, Mattiasson A, Bo K, Brubaker LP, De Lancey JO, Klarskov $\mathrm{P}$ et al (1996) The standardization of terminology of female pelvic organ prolapse and pelvic floor dysfunction. Am J Obstet Gynecol 175(1):10-17 